\title{
Removal of Intradural Extramedullary Schwannoma at Lumbar Level by Doing Laminoplasty in Paediatric Patient- A Case Report and Review of Literature
}

\author{
Rohit Thaker ${ }^{1}$, Arvind Gosai ${ }^{1}$, Pratik Shah ${ }^{1}$
}

\section{Abstract}

Intradural extramedullary tumour of thoracolumbar spine has been mainly treated with laminectomy till date. Other approach has been of treating this pathology by doing laminoplasty. Objective of this case presentation is that laminoplasty is better option for IDEM in selected cases. Laminectomy has been associated with many complications which can be avoided by doing laminoplasty such as postoperative spinal instability, epidural fibrosis, kyphotic deformity, excessive blood loss, hematoma invasion, progressive myelopathy, persistent back pain and prolonged hospital stay. So laminoplasty has clearly advantage compared to laminectomy in preserving posterior arch of the spine. Revision surgery is also easier when primary surgery has been done by laminoplasty. In our case of 13-year-old boy having Intradural extramedullary Schwannoma at L3 level, he was treated with flipping laminoplasty and tumour excision. At final follow up he was having complete clinical recovery and fully healed laminoplasty assessed with CT scan without any recurrence of tumour or any spinal deformity. It proves our purpose of doing laminoplasty with better outcome compared to laminectomy.

Keywords: Laminoplasty; Laminectomy; Intradural Extramedullary; Spinal Cord Tumour.

\section{Introduction}

Among the all CNS (central nervous system) tumours, spinal tumours comprise around 15\% [1]. And from them over 60\% of tumours found within the spinal canal are Intradural Extramedullary (IDEM) Spinal tumour [2]. Many different approaches have been described in literature to excise this type of tumours. Laminectomy has been gold standard approach for IDEM tumour excision but it has been associated with many complications. Laminoplasty has been also advocated for IDEM tumour excision in literature. It has been accepted very well in cervical IDEM surgery but literature also supports its use in thoracic and lumbar IDEM excision. Laminoplasty has edge over laminectomy in this type of surgery. Objective of our study is to prove that laminoplasty is an effective alternative to standard laminectomy with less chances of complications. Here we are presenting one such case of young patient who presented with lumbar IDEM lesion and was treated with laminoplasty approach. Patient was followed up thoroughly and had very good outcome.

Department of Spine Surgery, OrthoPlus Hospital,
Ahmedabad, Gujarat, India.
Address of correspondence :
Dr. Rohit Thaker,
Consultant Spine Surgeon, OrthoPlus Hospital,
Ahmedabad, Gujarat, India.
E-mail: thakerrohit@gmail.com

\section{Case History}

A 13-year-old boy presented to us with complaints of low back pain and bilateral lower limbs pain for 6 months. Patient had claudication symptoms and his walking was limited by 200 meters but no other neurological symptoms. He was treated with analgesics but symptoms were progressive. On clinical examination, there was mild midline tenderness over the lumbar spine with no obvious deformity. Neurological examination revealed normal tone and motor power in both lower limbs (5/5 MRC). Deep tendon reflexes were sluggish at ankle; plantar reflex was downward. There was no involvement of urinary bladder or bowel function. Sensory hypoesthesia was present below lumbar L3 level on the both sides. Patient was investigated in the form of radiographs and MRI scan. Radiology showed normal alignment of spine vertebrae. Magnetic resonance imaging (MRI) scan revealed well defined rounded soft tissue lesion at intraspinal intradural region along with cauda equina roots at level of L3 body and L3-4 disc level (Figure 1). It measures approximately $8^{*} 12^{*} 14 \mathrm{~mm}$ in size and causing moderate to significant canal stenosis with complete myelographic block at that level. It appears isointense in T1 images and heterogenous hyperintense in $\mathrm{T} 2$ images without any sign of calcification, haemorrhage or fat within lesion. Patient was having progressive symptoms so we decided to operate and planned to excise lesion by doing laminoplasty

2021 (C) Back Bone: The Spine Journal (The Official Journal Of "Spine Association Of Gujarat") | Available on www.backbonejournal.com | DOI:10.13107/bbj.2021.v02i01.020 This is an Open Access article distributed under the terms of the Creative Commons Attribution Non-Commercial License (http://creativecommons.org/licenses/by-nc/3.0) which permits unrestricted non-commercial use, distribution, and reproduction in any medium, provided the original work is properly cited. 


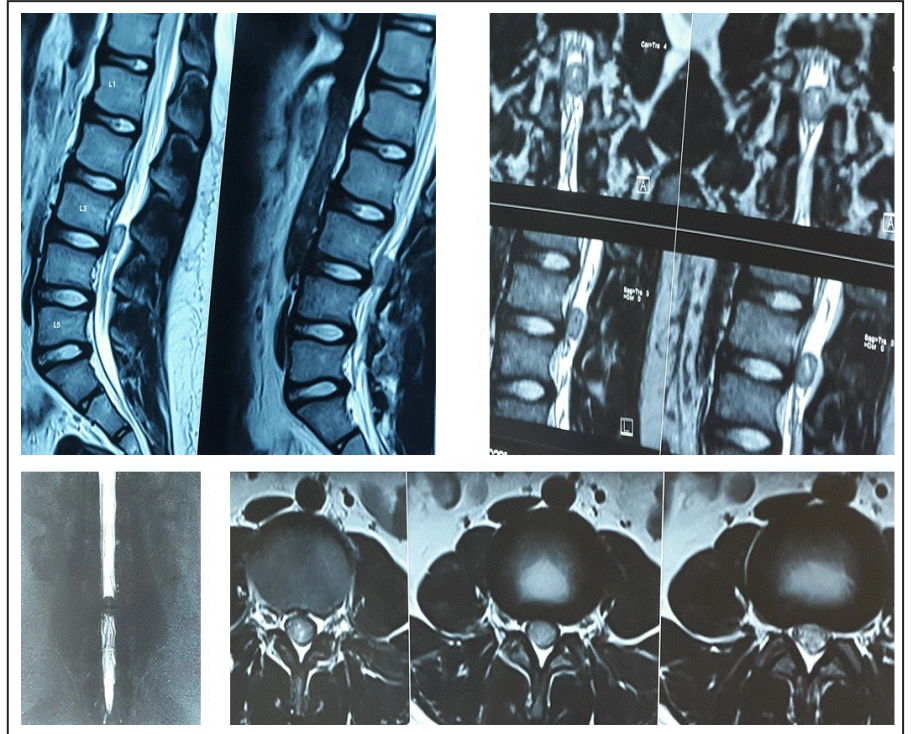

Figure 1: Pre-operative MRI suggestive of well-defined rounded soft tissue lesion at intraspinal intradural region along with cauda equina roots at level of L3 body and L3-4 disclevel.

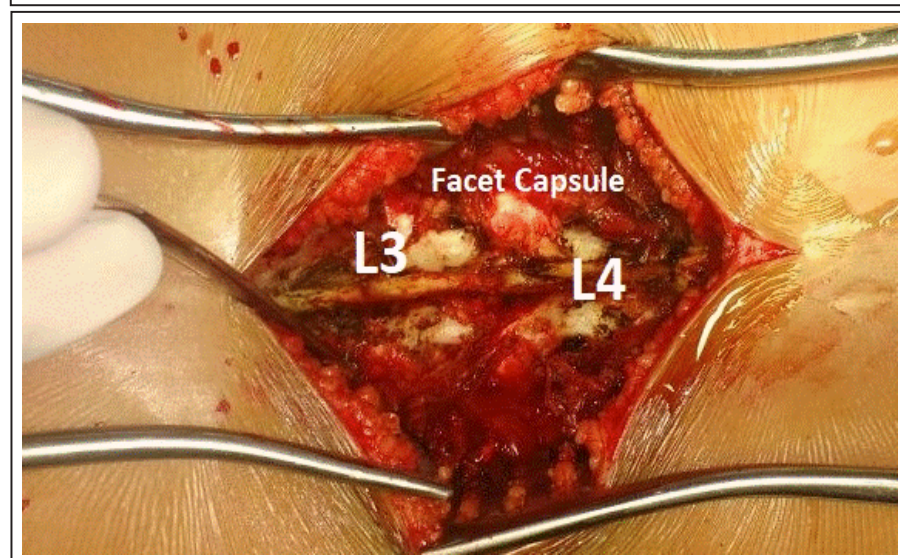

Figure 2: Standard mid-line approach with bilateral paraspina muscles subperiosteally erased, leaving the supra- and interspinous ligaments and the facet joint capsule intact.

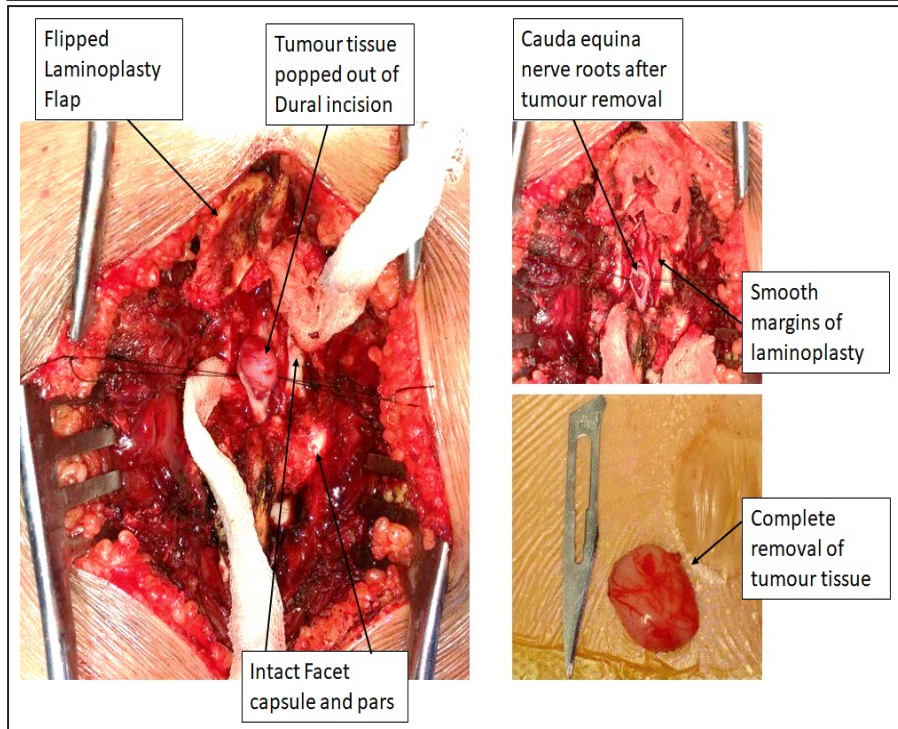

Figure 3: Excision of tumor tissue after doing laminoplasty

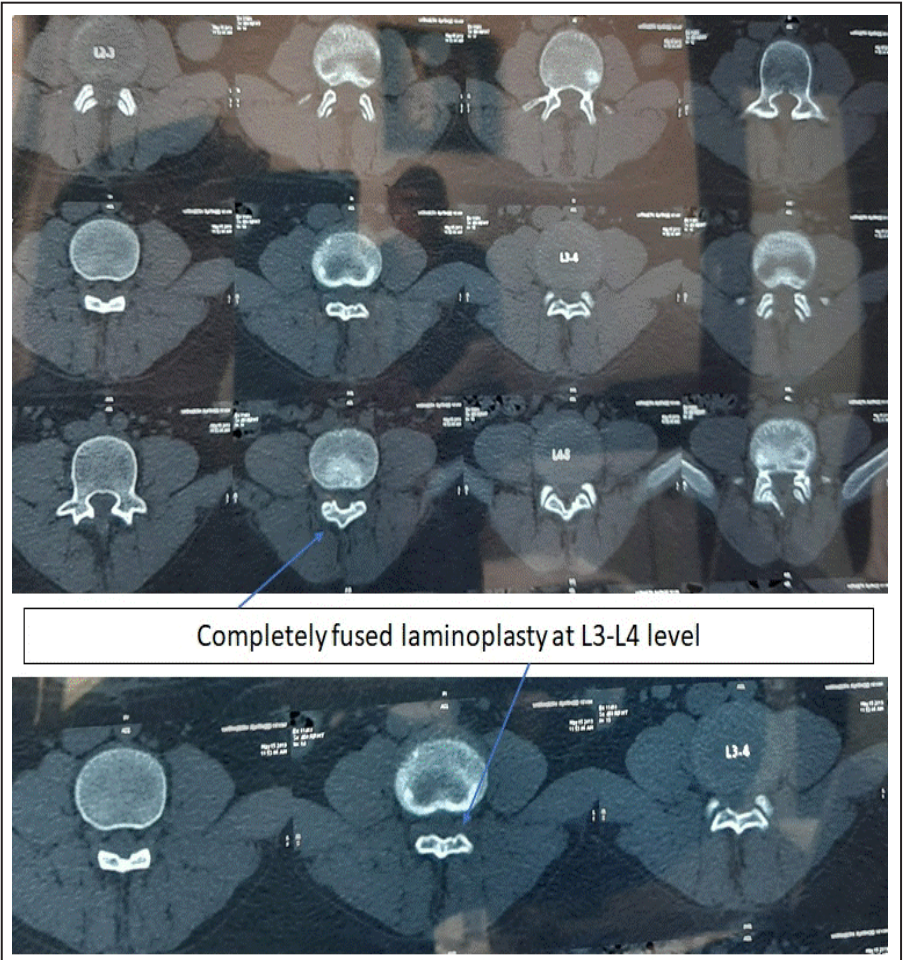

Figure 4: MRI study at 3 year follow up shows complete clearance of tumor tissue and no residual tumor or any recurrence. Laminoplasty site well healed.

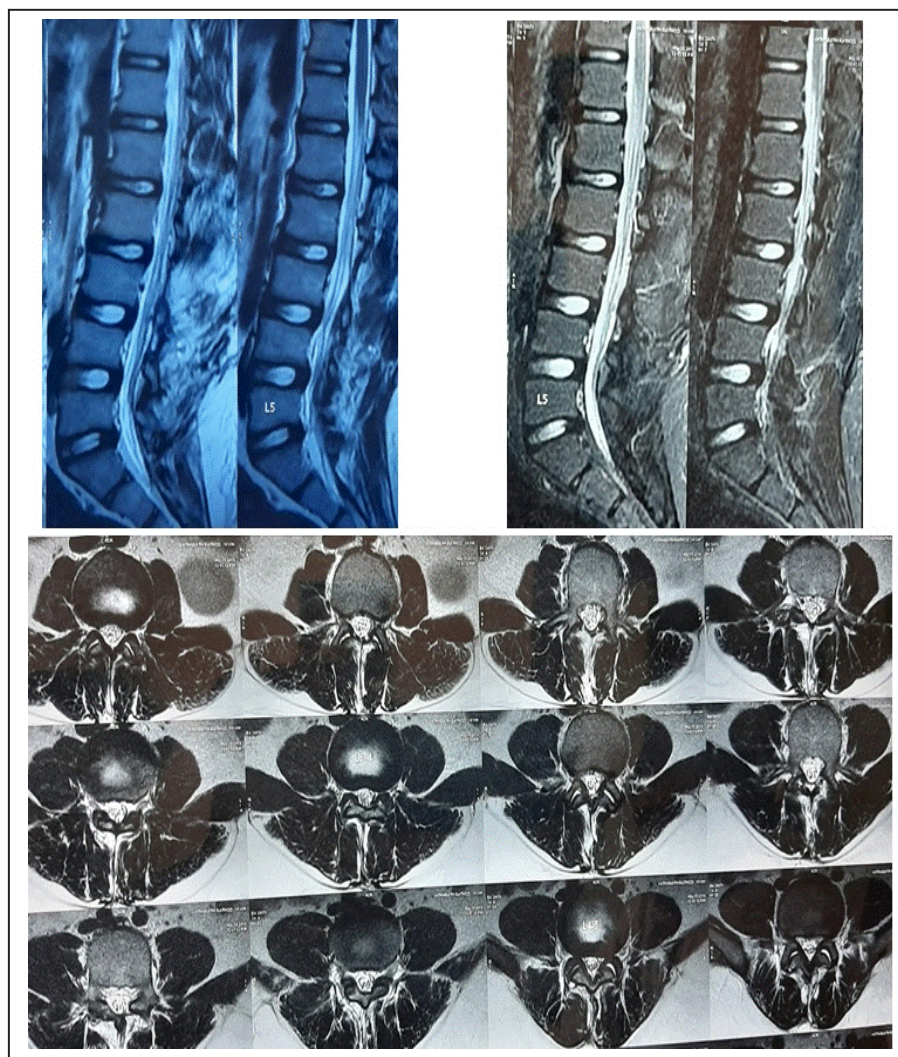

Figure 5: CT scan at 3 year follow up shows that osteotomy site of laminoplasty site was completely fused. 
rather than laminectomy. Pre-operative investigation and fitness was done and then planned for surgery.

Patient was positioned prone under general anaesthesia. A standard midline posterior approach was taken. Bilateral para $\neg$ spinal muscles were subperiosteally erased, leaving the supra- and interspinous ligaments and the facet joint cap $\urcorner$ sule intact (Figure 2). The lamina of L3 and L4 level were identified and confirmed under fluoroscopy. Osteotomy cuts were performed on both sides of the lamina just medial to the facet joint and pars with the help of high-speed burr in trapezoidal manner. An inferior cut was made through the superior half of the spinous process of the inferior vertebra and then whole osseo-ligamentous flap was elevated from distal end using blunt dissection with curette and broad dura retractor, leaving the superior attachment intact. The osseo-ligamentous flap was then flipped superolaterally, hinging over the superior in $\neg$ terspinous ligament to expose the spinal canal. Hemostasis was maintained using bipolar cautery and gauze piece with surgical patties. The dural sheath was incised carefully along the tumor bulge in the midline. The tumor mass was excised en bloc by blunt dissection using a Penfield dissector (Figure 3). The dural sheath was sutured with 4-0 non-absorbable suture. Any sharp bony prominences on the laminoplasty flap margins were smoothened. The posterior arch was then recreated by repositioning the flap that was secured using Ethibond 2-0 suture through the flap and remaining inferior spinous process. Closure was done in layers and positive drain were kept. Tumour tissue which was removed, sent for histopathological examination (Biopsy). Postoperative period was uneventful and neurological assessment was done. Patient was having same neurology as pre-op. Lumbo-Sacral Brace was given and patient was mobilised after suture removal. Post-operative rehabilitation was done by doing active assisted physiotherapy of both lower limbs and supported walking for 4 weeks and then walking without support was started.

Follow up was done at 15 days, 3, 6 and 12 months and last follow up was done at 3 years. Patient was assessed clinically and radiologically. Clinically patient was improved within one month and his claudication symptoms were improved completely. Histopathological examination (Biopsy) was suggestive of Schwannoma. At final follow up, MRI and CT scan were done. MRI shows complete clearance of tumour (Figure 4) and CT scan shows that laminoplasty was completely fused (Figure 5).

\section{Discussion}

For the excision of IDEM tumour, many different surgical techniques have been described in the literature but laminectomy has been the conventional approach. Problem with the laminectomy is that it has been associated with several potential complications such as postoperative spinal instability, epidural fibro $\neg$ sis, kyphotic deformity, excessive blood loss, hematoma invasion, progressive myelopathy, persistent back pain and prolonged hospital stay $[3,4,5]$. As there is chance of recurrence in IDEM tumours, revision surgery is also an issue with laminectomy as the dura is left without bony cover, making exposure during the repeat surgery a challenge [6].

Laminoplasty has also been used in thoracic and lumbar spinal surgery, especially in cases of intradural tumour and lumbar canal stenosis (LCS). It has advantage of preserving posterior arch of the spine, protect paraspinal muscles, and prevent postoperative instability, epidural adhesion and kyphotic deformities. Laminoplasty procedure for excision of intradural tumours it is relatively easy compare to LCS surgery as it is not necessary to enlarge the spinal canal or lateral recess, nor to excise the ligamentum flavum for decompression of the cauda equina and nerve roots in tumour cases. In case of recurrence of IDEM, the technique of laminoplasty makes revision surgery much easier, as it is safer to expose the dura after this type of surgery than after traditional laminectomy, because it preserves a relatively normal posterior bony structure and there is no epidural scar; hence laminoplasty may be a suitable option for this type of patient [7]. Many different type of laminoplasty techniques has been reported in literature but in the recent literature, the most popular method of osteotomy has been to make gutters just medial to the facet joints to avoid instability after surgery, and to remove the laminae in a trapezoid manner to prevent them sinking into the spinal canal [8].

Papagelopoulos et al. reported 14-year follow-up results after multilevel lumbar or thoracolumbar total laminectomy for removal of benign intraspinal tumours [9]. Spinal column deformity occurred in $33 \%$ of the children and adolescents and in $8 \%$ of the young adults. Spondylolisthesis occurred in $16.6 \%$ of the children and adolescents and in $8 \%$ of the young adults. Yeh et al. found that laminectomy is associated with a worse alignment at the thoracic-thoracolumbar region, while laminoplasty reduces this risk in children [10]. However, Wiedemayer's results show that laminoplasty does not prevent worsening of any pre-existing deformity [8].

Time taken for fusion at the site of laminoplasty ranges from 1.9 months to 5 months according to the literature. It is important to re-attach laminae to its anatomical site to heal it very well and give adequate spinal mechanical stability by preserving ligament attachment and prevent postoperative epidural scarring. Yücesoy and Crawford reported that, using inverse laminoplasty, bony fusion of the incision line of the laminae was seen in only one third of patients who were monitored for more than 2 years [11]. Wiedemayer et al. reconstructed the laminar roof with miniplates to the anatomical site in 79 cases (laminae). In his follow-up results, $11.3 \%$ laminae achieved unilateral fusion while $2.6 \%$ developed no osseous bridging of 54 | Back Bone: The Spine Journal | Volume 1 | Issue 1 | April-September 2021 | Page 52-55 
the osteotomy sites on either side [8]. In study by X Liu et al. the time to achieve fusion of the laminae averaged 4.5 months, among them $10.6 \%$ achieved unilateral fusion of the incision line in the laminae, while in no case was bilateral non-union observed [7]. There are two shortcomings of this technique as described by X Liu et al: (i) after laminoplasty, external fixation for an extended period of time is necessary, which prolongs the period of bed rest and prevents the patients from undergoing early mobilization and rehabilitation; (ii) multiple laminoplasty (at more than three levels) for the treatment of IDEM tumours should not be considered in patients with lumbar or cervical tumours, in order to avoid spinal instability, especially in young patients. Fixing the laminae, spinous processes and ligaments with sutures can achieve satisfactory primary stability and high fusion rates for resected laminae. Additional instrumentation may be necessary in greater than three-level laminoplasty [7]. There has not been any specific criteria for excision of long segment intradural lesion that it can only be removed with laminectomy and laminoplasty can not be done. As reported by Onyia et al., retrospective evaluation in their study demonstrates that laminoplasty not to be more or less likely to have any better functional outcome or need for revision compared to laminectomy in the resection of longsegment intradural lesions. A quality randomized controlled study on a much larger scale will be required to validate this finding [12].

As it has been concluded by Ramesh et al [6] this type of flipped reposition laminoplasty is an excellent technique for the excision of IDEM tumours of the thoracolumbar spine and it provides adequate exposure of spinal canal as well as ad $\neg$ ditional stability to spine. Limitation of this case is that it is single case study and conclusion should be drawn from multiple case series or well-designed prospective study in which all parameters of pre and post-operative spinal alignment like kyphosis and lordosis angle should be studied, only than it can be reliably proved that laminoplasty is superior than laminoplasty in treating IDEM.

Key Message: Laminoplasty is safe and advantageous over laminectomy in removal of Intradural Extramedullary tumour with less complications and if revision surgery needed, it is much safer in laminoplasty cases.

\section{References}

1. Arnautovic K, Arnautovic A. Extramedullary intra $\neg$ dural spinal tumors: a review of modern diagnostic and treatment options and a report of a series. Bosn $J$ BasicMed Sci2009;9 Suppl 1:40-5.

2. Aghayev K, Vrionis F, Chamberlain MC. Adult in $\neg$ tradural primary spinal cord tumors. JNatl Compr Canc Netw 2011;9:434-47.

3. Iida $Y$, Kataoka $O$, Sho T, et al. Postoperative lumbar spinal instability occurring or progressing secondary to laminectomy. Spine (Phila Pa 1976) 1990;15:11869.

4. Mayfield FH. Complications oflaminectomy. Clin Neurosurg 1976;23:435-9.

5. Yasuoka S, Peterson HA, MacCarty CS. Incidence of spinal column deformity after multilevel laminecto $\neg m y$ in children and adults. J Neurosurg 1982;57:4415 .

6. Kumar R, Debbarma I, Boruah T, et al. Flipped Reposition Laminoplasty for Excision of Intradural Extramedullary Tumors in the Thoracolumbar Spine: A Case Series of 14 Patients. Asian Spine J. 2020;14(3):327-335. doi:10.31616/asj.2019.0034

7. Liu XY, Zheng YP, Li JM. Laminoplasty for the treat 7 ment of extramedullary intradural tumors in the tho $\neg$ racic and lumbar spine: greater than two-year follow-up. Orthop Surg 2009;1:275-9.
8. Wiedemayer H, Sandalcioglu IE, Aalders $M$, et al. Reconstruction of the laminar roof with miniplates for a posterior approach in intraspinal surgery: technical considerations and critical evaluation of follow-up results. Spine, 2004, 29: E333-E342.

9. Papagelopoulos PJ, Peterson HA, Ebersold MJ, et al. Spinal column deformity and instability after lumbar or thoracolumbar laminectomy for intraspinal tumors in children and young adults. Spine, 1997, 22:442-451.

10. YehJS, Sgouros S, Walsh AR, et al. Spinal sagittal malalignment following surgery for primary intramedullary tumours in children. Pediatr Neurosurg, 2001, 35: $318-324$.

11. Yücesoy $K, C r a w f o r d ~ N R$. Increase in spinal canal area after inverse laminoplasty: an anatomicalstudy. Spine, 2000, 25: 2771-2776.

12. Onyia, Chiazor \& Menon, Sajesh. (2018). Laminectomy Versus Laminoplasty in the Surgical Management of Long-Segment Intradural Spinal Tumors: Any Difference in Neurological Outcomes?. Asian Journal of Neurosurgery. 13. 1128. 10.4103/ajns.AJNS_67_18.
Conflict of Interest: NIL Source of Support: NIL

\section{How to Cite this Article}

Thaker R, Gosai A, Shah P $\mid$ Removal of Intradural Extramedullary Schwannoma at Lumbar Level by Doing Laminoplasty in Paediatric Patient- A Case Report and Review of Literature BackBone: The Spine Journal | April-September 2021;2(1):52-55. 